\title{
Numerical Investigation on Heat Transfer in Confined Impinging Slot Jets with Nanofluids in Partially Filled Configuration of Metal Foam
}

\author{
Bernardo Buonomo, Furio Cascetta, Anna di Pasqua, Oronzio Manca*, Sergio Nappo \\ Università degli Studi della Campania “Luigi Vanvitelli”, Dipartimento di Ingegneria, Via Roma 29, \\ 81031 Aversa, Italy
}

\begin{abstract}
In this paper a numerical investigation on mixed convection in confined slot jets impinging on a partially filled configuration of porous medium by considering pure water or $\mathrm{Al}_{2} \mathrm{O}_{3}$ /water based nanofluids is described. A two-dimensional model is developed and different Peclet numbers are considered. Rayleigh numbers is imposed equal to 30000 . The particle volume concentration ranges from $0 \%$ to $4 \%$ and the particle diameter is assumed equal to 20,30 and $80 \mathrm{~nm}$. The target surface is heated at constant temperature value, calculated according to the value of Rayleigh number. The distance of the target surface is five times greater than the slot jet width. Three different values of the ratio between the total system length and metal foam length are considered. A single-phase model approach is employed in order to describe the nanofluid behaviour while the hypothesis of non-local thermal equilibrium is assumed to simulate the thermal behaviour in the metal foam. The foam is characterized by a number of pores per inch equal to 5, 10, 20 and 40 and a porosity around 0.90 . The aim of the paper consists to study the thermal and fluid-dynamic behaviour of the impinging jet system with nanofluids. Results show increasing values of the Nusselt number for increasing values of Peclet number and nanoparticle concentration. In addition, the ratio between the thermal and pumping power is evaluated to find a trade-off between the increase of heat transfer and pressure drop.
\end{abstract}

\section{Introduction}

Nowadays more and more efficient systems are built to improve the heat exchange in different applications such as in automotive, aerospace, solar energy heaters, electronic and process industry. Among the various solutions that can guarantee a good cooling system one of the best is the confined impinging jets. Furthermore, the use of jets combined with the application of nanofluids as a working fluid brings a further improvement in terms of thermal efficiency with respect to the pure water. For their properties, such as high cooling effectiveness, uniformity and controllability, the impinging slot jet are finding wide fields of application. Furthermore, the use of porous media inside impinging jets is becoming a

\footnotetext{
* Corresponding author: oronzio.manca@unicampania.it
} 
widely used technique because its employment allows to obtain a further improvement in terms of heat transfer efficiency.

Amjadian et al. [1] carried out an experimental investigation on a round impinging jet on a hot disk surface with constant heat flux with the application of $\mathrm{Cu}_{2} \mathrm{O}$-water nanofluid. The nanoparticle concentrations ranged from $0.03 \mathrm{wt} \%$ to $0.07 \mathrm{wt} \%$ while the Reynolds number was variable from 7330 to 11,082 . The results showed that near the heated surface the heat transfer coefficient increased for the turbulence of the fluid flow. In addition, the best improvement in terms of heat transfer was obtained for the nanofluid at $0.07 \mathrm{wt} \%$. A numerical investigation on a two-dimensional confined slot jet with $\mathrm{Cu}$-water nanofluid impinging on a hot moving isothermal plate was accomplished by Datta et al. [2]. The effect of several parameters such as plate velocity, Reynolds number, nanoparticles volume concentration and geometric ratio of the flow were investigated. For $\operatorname{Re}=20000$ an increase in terms of Nusselt number equal to $17 \%$ and $31 \%$ was obtained for nanoparticles volume concentration of $3 \%$ and $5 \%$, respectively. The fluid flow and convective heat transfer were governed by plate motion when its velocity increased. The average Nusselt number, with geometrical parameters, Reynolds number and nanoparticles concentration fixed, showed an initial decrease and then increased quickly as the plate velocity was increased. Alabdaly et al. [3] accomplished a numerical study on the heat transfer improvement using a confined slot impinging jet with $\mathrm{SiO}_{2}$-water nanofluid. Two circular ribs were located on the bottom of the target surface at right and left of the stagnation point. Results show that the average Nusselt number, performance factor, total entropy generation and friction factor increase with increasing nanoparticles volume concentration. Furthermore, the best thermal and fluid dynamic performance factor was around 1.89 for Reynolds number equal to 1000 , nanoparticles volume fraction of $4 \%$, the rib height of 0.1 and the rib position of 2. A numerical analysis on single- and two-phase models of nanofluid in a confined impinging jet was carried out by Abdelrehim et al. [4]. Reynolds number ranged from 100 to 400 and the jet height ratio varied from 0.5 to 4 . The working fluid was water- $\mathrm{Al}_{2} \mathrm{O}_{3}$ nanofluid with a volume fraction concentration $(\varphi)$ equal to $0 \%, 1 \%$ and $4 \%$. The results showed that the Nusselt number increased with increasing both Re and $\varphi$. The two-phase model expected a higher Nusselt number respect to the single-phase model due to the presence of the heat exchange term in the energy equation. The average Nusselt number presented the maximum improvement equal to $150 \%$ in corresponding to the two-phase model and $\varphi=4 \%$ and $\mathrm{H} / \mathrm{W}=4$. Furthermore, the pumping power ratio was 2.99 times higher than the value obtained for pure water in single-phase for $\varphi=4$. In twophase model, this ratio was higher only 1.49 times. An experimental analysis on heat transfer characteristics of hybrid nanofluid impinging jets was accomplished by Sun et al. [5]. The working fluids used were Silver (Ag)-multiwall carbon nanotube/water hybrid nanofluids. The experimental investigation studies both conventional impinging jets (CIJs) and swirling impinging jets (SIJs). The results showed that the thermal conductivity of Agmultiwall carbon nanotube/water hybrid nanofluids was greater than the one for multiwall carbon nanotube nanofluids. The heat transfer coefficient of $0.01 \%$ Ag-multiwall carbon nanotube/water hybrid nanofluids increased by $29.45 \%$ and $29.37 \%$ respect to the pure water, respectively. Moreover, the heat transfer coefficient of $0.01 \%$ multiwall carbon nanotube/nanofluids increased by $20.42 \%$ and $13.35 \%$, respectively, in the conventional impinging jets and swirling impinging jets. In the CIJs and SIJs, the heat transfer coefficient of $0.05 \%$ Ag-multiwall carbon nanotube/water hybrid nanofluid increased by $116.67 \%$ and $120.53 \%$ respect to the pure water, respectively.

Izadi et al. [6] carried out an analytical study on CPU cooling by nanofluid in metal foam. The impinging jet was made up of nanofluids under effects of magnetic field. The authors solved nonlinear ordinary differential equations (ODEs) obtained with the use of similarity variables in order to convert the original partial differential governing equations. 
The system was constituted by a rectangular box of metal foam with the bottom surface that represented the hot CPU part and an imping jet of water-alumina nanofluid was present in the top. The results showed that the increase of Darcy number improved the heat transfer, while an opposite behaviour was detected increasing the aspect ratio and Eckert number. Moreover, the Nusselt number slightly decreased as increased the Hartman number for low porosities; but an opposite trend was noted at high porosity. Another numerical analysis on the thermal behaviour of a heat sink with metal foam and impinging jet was carried out by Izadi et al. [7]. Hydrogen, air and $\mathrm{Cu}$-water nanofluid are assumed as working fluids. Results showed that with the increase of the porosity the velocity and temperature profiles decreased. Furthermore, the increase of Darcy number caused an increase in terms of heat transfer rate. The temperature profile, instead, increased with the increase of the distance from the symmetry axis and, at the same time, the velocity profile decreased. In conclusion, the impinging jet characterized by a non-uniform velocity profile with decreasing slope represents the best condition in terms of heat transfer rate.

A numerical investigation on a cylindrical heat sink with porous medium and air/nanofluid imping jet was accomplished by Siavashi et al. [8]. The results showed that the increment of Re caused the increase of heat transfer rate. Furthermore, the increase of porosity reduced the shear stress on the wall and also decreased the temperature profile. In conclusion, the increase of nanoparticles concentration enhances the values of the average Nusselt number.

In this work a numerical analysis on mixed convection in confined slot jets impinging on a partially filled configuration of aluminium foam with $\mathrm{Al} 2 \mathrm{O} 3 /$ water based nanofluids characterized by particle volume concentrations from $0 \%$ to $4 \%$ and the particle diameters equal to 20,30 and $80 \mathrm{~nm}$ is illustrated. A two-dimensional model is studied with different Peclet number values. Rayleigh numbers is considered equal to 30000 . The target surface is characterized by a constant temperature value, estimated according to the value of Rayleigh number. Three different values of ratio between the total system length and the metal foam length, equal to 1, 3.5 and 7, are examined. The Local Thermal Non-Equilibrium (LTNE) model is applied to describe the behaviour of the metal foams characterized by the number of pores per inch (PPI) equal to 5, 10, 20 and 40 and a porosity around 0.90 . Results are reported in terms of Nusselt number and pressure drop for several ratios between the total system and the metal foam length, for different nanoparticle diameter values and for different characteristics of metal foams. In conclusion, the ratio between the thermal and pumping power is estimated to obtain a compromise between the increment of heat transfer and pressure drop.

\section{Physical model and governing equations}

The physical model is a confined impinging slot jet in a porous medium with nanofluids. The two-dimensional model depiction of the analysed system is showed in Figure 1. The impinging slot jet is made up of a height $H$ equal to $30 \mathrm{~mm}$ and a length $2 L$ of $420 \mathrm{~mm}$. The width of jet, indicted as $2 W$, is equal to $6 \mathrm{~mm}$ and the length of the heated part of the wall, $2 L_{h w}$, is $60 \mathrm{~mm}$. The local volume averaging process is applied at the microscopic flow equations as indicated in [9] and [10] to replicate the thermal and fluid dynamic performance inside the domain. The variables of the equations are evaluated estimating the average of the local variables in a particular volume, called Representative Elementary Volume (REV), using the local volume averaging technique. The aluminum foam, the employed porous medium of the analysis, is assumed homogeneous and isotropic and the properties of the fluid and solid phases are considered constant. The DarcyForchheimer-Brinkman and LTNE models are assumed to describe the presence of the metal foam. Using the hypothesis above indicated, the governing equations are: 


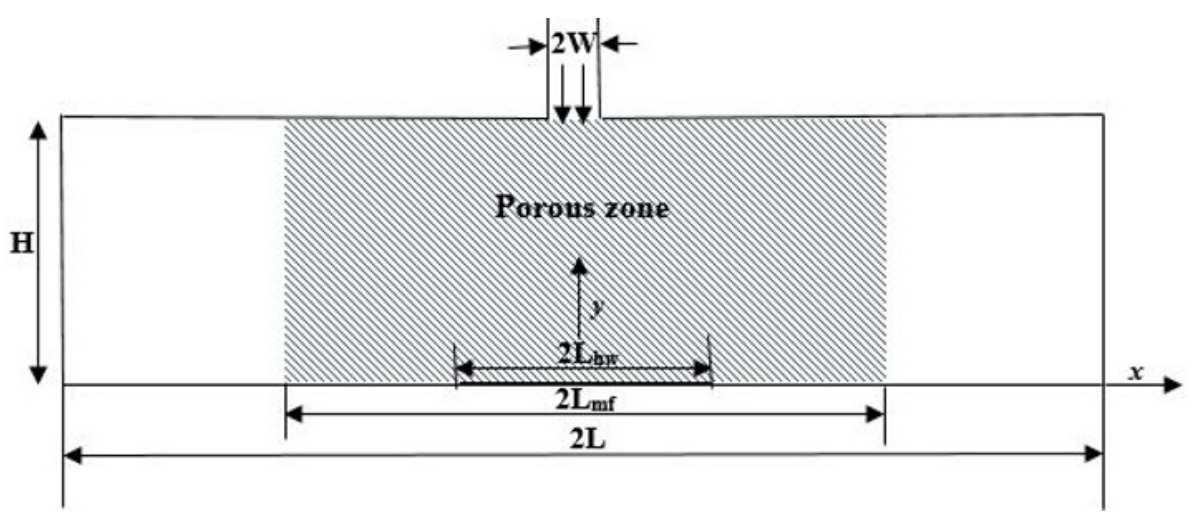

Fig. 1. Physical Domain

-Continuity equation

$$
\frac{\partial u}{\partial x}+\frac{\partial v}{\partial y}=0
$$

-x- momentum equation

$$
\begin{aligned}
& \rho_{n f}\left(\frac{u}{\varepsilon^{2}} \frac{\partial u}{\partial x}+\frac{v}{\varepsilon^{2}} \frac{\partial u}{\partial y}\right)=-\frac{\partial p}{\partial x}+\frac{\mu_{n f}}{\varepsilon}\left(\frac{\partial^{2} u}{\partial x^{2}}+\frac{\partial^{2} u}{\partial y^{2}}\right)-\delta\left(\frac{\mu_{n f}}{K} u+\frac{C_{F}}{K^{1 / 2}} \rho_{n f} \sqrt{u^{2}+v^{2}} u\right) \\
& +\rho_{n f} g \beta_{n f}\left(T_{n f}-T_{0}\right)
\end{aligned}
$$

-y-momentum equation

$$
\rho_{n f}\left(\frac{u}{\varepsilon^{2}} \frac{\partial v}{\partial x}+\frac{v}{\varepsilon^{2}} \frac{\partial v}{\partial y}\right)=-\frac{\partial p}{\partial y}+\frac{\mu_{n f}}{\varepsilon}\left(\frac{\partial^{2} v}{\partial x^{2}}+\frac{\partial^{2} v}{\partial y^{2}}\right)-\delta\left(\frac{\mu_{n f}}{K} v+\frac{C_{F}}{K^{1 / 2}} \rho_{n f} \sqrt{u^{2}+v^{2}} v\right)
$$

where $\varepsilon$ is the porosity, $\rho_{n f}$ and $\mu_{n f}$ are nanofluid density and viscosity, $u$ and $v$ are the velocity components in Cartesian coordinates, $K$ and $C_{F}$ are the permeability and inertial coefficient of the metal foam, respectively.

-Fluid phase energy equation

$$
\left(\rho c_{p}\right)_{n f}\left(u \frac{\partial T_{n f}}{\partial x}+v \frac{\partial T_{n f}}{\partial y}\right)=\varepsilon k_{n f}\left(\frac{\partial^{2} T_{n f}}{\partial x^{2}}+\frac{\partial^{2} T_{n f}}{\partial y^{2}}\right)+\delta h_{s f} \alpha_{s f}\left(T_{s}-T_{n f}\right)
$$

-Solid phase energy equation

$$
(1-\varepsilon) k_{s}\left(\frac{\partial^{2} T_{s}}{\partial x^{2}}+\frac{\partial^{2} T_{s}}{\partial y^{2}}\right)-\delta h_{s f} \alpha_{s f}\left(T_{s}-T_{n f}\right)=0
$$


in which $c_{p}$ is the nanofluid specific heat, $k_{n f}$ and $k_{s}$ are the fluid phase and solid matrix thermal conductivity, $T_{n f}$ and $T_{s}$ are the temperature of fluid phase and solid phases of metal foam, respectively. The terms $\alpha_{s f}$ and $h_{s f}$ are the specific surface area density and the interfacial heat transfer coefficient between the fluid phase and solid matrix, due to the LTNE model. Finally, the governing equations (1)-(5) are used for both porous and clean region, where $\delta=1$ and $0<\varepsilon<1$ for porous zone, $\delta=0$ and $\varepsilon=1$ for clean zone.

The evaluation of the parameters $K$ and $C_{F}$ has been carried out by means of the correlations of Bhattacharya et al. [11], while the relationships of Calmidi et al. [12] are used to estimate the values of $\alpha_{s f}$ and $h_{s f}$. $[11,12]$

The key parameters of the metal foams, used in this study, are listed in the Table 1.

Table 1. Parameters of the used aluminum foams

\begin{tabular}{|c|c|c|c|}
\hline PPI & $\boldsymbol{\varepsilon}$ & $\boldsymbol{K}\left[\mathrm{m}^{2}\right]$ & $\boldsymbol{C} \boldsymbol{F}$ \\
\hline 5 & 0.905 & $1.7 \mathrm{e}^{-7}$ & 0.078 \\
\hline 10 & 0.8991 & $9.4 \mathrm{e}^{-8}$ & 0.068 \\
\hline 20 & 0.9005 & $9.0 \mathrm{e}^{-8}$ & 0.088 \\
\hline 40 & 0.9132 & $5.3 \mathrm{e}^{-8}$ & 0.084 \\
\hline
\end{tabular}

The present work investigates the laminar flow and the corresponding convective heat transfers of water-based/ $\mathrm{Al}_{2} \mathrm{O}_{3}$ nanofluids under steady-state conditions. The particle volume concentrations ranged from $0 \%$ to $4 \%$.

A single-phase model approach has been adopted in order to describe the nanofluid behavior. The density and the specific heat are evaluated with the expressions of the work [13].

The choice of the correlations to calculate the nanofluid properties has been made by means of the work of Sekrani et al. [14]. For the dynamic viscosity of the nanofluid, the correlation employed is [15]:

$$
\frac{\mu_{n f}}{\mu_{b f}}=1+39.11 \phi+533.9 \phi^{2}
$$

The correlation of [16] is used to evaluate the nanofluid thermal conductivity:

$$
\frac{k_{n f}}{k_{b f}}=\left[\frac{k_{n p}(1+2 \alpha)+2 k_{b f}-2 \phi\left(k_{b f}-k_{n p}(1-\alpha)\right)}{k_{n p}(1+2 \alpha)+2 k_{b f}+\phi\left(k_{b f}-k_{n p}(1-\alpha)\right)}\right]
$$

where $\alpha=2 R_{b} k_{b f} / d_{n p}$ is the particle Biot number and $R_{b}=0.77 \times 10^{-8} \mathrm{~m}^{2} \mathrm{KW}^{-1}$ is the interface thermal resistance.

The nanofluid thermal expansion coefficient $\beta$ is evaluated as [13]:

$$
\frac{\beta_{n f}}{\beta_{b f}}=\left[\frac{1}{1+\left(\frac{1-\phi}{\phi}\right) \frac{\rho_{b f}}{\rho_{n p}}} \frac{\beta_{n p}}{\beta_{b f}}+\frac{1}{1+\left(\frac{\phi}{1-\phi}\right) \frac{\rho_{n p}}{\rho_{b f}}}\right]
$$


The properties of the alumina nanoparticles are set to: $\rho_{n p}=3880 \mathrm{~kg} \mathrm{~m}^{-3}, c_{p n p}=729 \mathrm{~J} \mathrm{~kg}^{-}$ ${ }^{1} \mathrm{~K}^{-1}, k_{n p}=42 \mathrm{~W} \mathrm{~m}^{-1} \mathrm{~K}^{-1}$.

The diameter of nanoparticles is assumed equal to 20,30 and $80 \mathrm{~nm}$. As the diameter of the nanoparticles changes, the only thermophysical quantity that changes is the nanofluid thermal conductivity.

The thermophysical properties of the nanofluids employed in the analyses are listed in Table 2 .

Table 2. Properties of the working fluids

\begin{tabular}{|c|c|c|c|c|c|c|c|}
\hline \multirow[t]{2}{*}{$\phi$} & \multirow[t]{2}{*}{$\begin{array}{c}\boldsymbol{\rho} \\
{\left[\mathrm{kg} / \mathrm{m}^{3}\right]}\end{array}$} & \multirow[t]{2}{*}{$\begin{array}{c}\boldsymbol{\mu} \\
{[\mathrm{Pa} \mathrm{s}]}\end{array}$} & \multicolumn{3}{|c|}{$\begin{array}{c}\boldsymbol{k} \\
{[\mathrm{W} /(\mathrm{m} \mathrm{K})]}\end{array}$} & $\begin{array}{c}c_{p} \\
{[\mathrm{~J} /(\mathrm{kg} \mathrm{K})]}\end{array}$ & $\boldsymbol{\beta}[1 / \mathrm{K}]$ \\
\hline & & & $\begin{array}{c}\boldsymbol{d}_{\boldsymbol{n} \boldsymbol{p}}=20 \\
\mathrm{~nm}\end{array}$ & $\begin{array}{c}\boldsymbol{d}_{\boldsymbol{n} \boldsymbol{p}}=30 \\
\mathrm{~nm}\end{array}$ & $\begin{array}{c}\boldsymbol{d}_{\boldsymbol{n} \boldsymbol{p}}= \\
80 \mathrm{~nm}\end{array}$ & & \\
\hline $0 \%$ & 998.2 & $1.0 \mathrm{e}^{-3}$ & \multicolumn{3}{|c|}{0.600} & 4182 & $2.1 \mathrm{e}^{-3}$ \\
\hline $1 \%$ & 1027 & $1.4 \mathrm{e}^{-3}$ & 0.605 & 0.607 & 0.613 & 4052 & $2.0 \mathrm{e}^{-3}$ \\
\hline $4 \%$ & 1113 & $3.4 \mathrm{e}^{-3}$ & 0.620 & 0.630 & 0.651 & 3701 & $1.8 \mathrm{e}^{-3}$ \\
\hline
\end{tabular}

The boundary conditions of the problem are the following: the left edge is symmetric, the upper and bottom edges are adiabatic wall, the heated wall is an isothermal edge. The impinging jet is characterized by a temperature $T_{0}$ equal to $293 \mathrm{~K}$ and a velocity equal to $u_{0}$. The outflow condition is imposed to the exit. The reference length is the width of the jet $W$ for the dimensionless number.

In this study, different Peclet numbers are assumed. Peclet number $P e$ evaluated as:

$$
P e=\operatorname{RePr}_{n f}=\frac{\rho_{n f} c_{p, n f} u_{0} W}{k_{n f}}
$$

The impinging jets velocities $u_{0}$ are evaluated from the definition of $P e$. Rayleigh number $R a$ defined as:

$$
R a=G r P r_{n f}=\frac{\rho_{n f}^{2} c_{p n f} \beta_{n f} \Delta T W^{3}}{\mu_{n f} k_{n f}}
$$

The assigned temperatures of the impinged heated wall are calculated by means of $R a$.

The average total Nusselt number $\overline{N u_{\text {tot }}}$ evaluated as:

$$
\overline{N u_{t o t}}=\overline{N u_{n f}}+\overline{N u_{s}}
$$

in which $\overline{N u_{n f}}=\overline{h_{n f}} W / k_{n f}$ and $\overline{N u}=\overline{h_{s}} W / k_{n f}$ are the fluid phase and solid matrix Nusselt number of the metal foam, respectively.

\section{Numerical model and governing equations}

The governing equations are solved with the finite volume method. The numerical investigations are executed by means of the code Ansys-Fluent 15.0. The SIMPLE algorithm is carried out for the pressure-velocity coupling; the least square cell is applied 


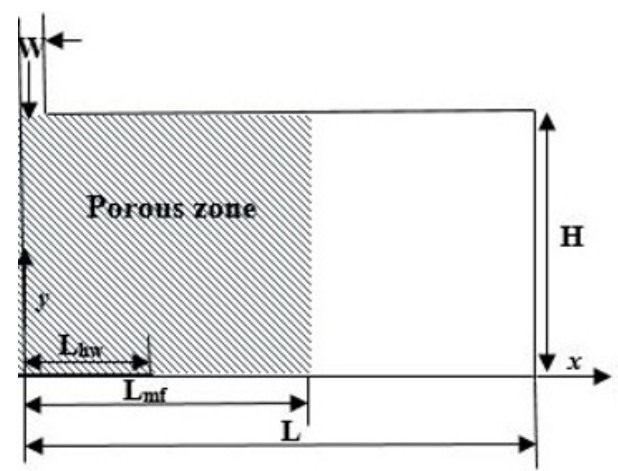

Fig. 2. Numerical Domain

for estimating the gradient evaluation for the spatial discretization. The PRESTO algorithm is applied to obtain the pressure calculation; the second order upwind scheme is employed for energy and momentum equations. Convergence criteria are equal to $10^{-5}$ for the continuity and the velocity components while for the energy equal to $10^{-8}$.

The numerical domain is showed in the Figure 2.

The grid is made up of rectangular cells. Three different types of grids were investigated to find an independent solution from the mesh. They are constituted by 52800 cells, 105600 cells and 211200 cells, respectively. In corresponding of a $\phi=4 \%, d_{n p}=20 \mathrm{~nm}, P e=750$, $L_{m f} / L_{h w}=7$ and of a metal foam of $20 \mathrm{PPI}$, the evaluation of average $\overline{N u_{\text {tot }}}$ on the heated wall, as showed in Table 3, points out that the grid with 105600 cells had $0.2 \%$ error than the mesh with 211200 cells. The grid adopted for the computational was the one with 105600 elements because a good compromise between solution accuracy and convergence time is obtained.

The grids for the other configurations are built up with the same criteria of the construction of the mesh for the ratio $L_{m f} / L_{h w}$ equal to 7 .

Table 3. Grid Independence

\begin{tabular}{|c|c|c|}
\hline Cells Number & $\overline{N u_{\text {tot }}}$ & \% error \\
\hline 52800 & 20.03 & $0.8 \%$ \\
\hline 105600 & 20.15 & $0.2 \%$ \\
\hline 211200 & 20.19 & ---- \\
\hline
\end{tabular}

\section{Results and discussion}

The thermal and fluid dynamic analyses are carried out for three different Peclet values equal to $350,750,1500$, and for Rayleigh number equal to 30000 .

For brevity, the average Nusselt number results, evaluated on the impinging heated wall, for different Peclet number values and for several ratio $L_{m f} / L_{h w}$ are showed in Figure 3 only for $5 \mathrm{PPI}, \phi=4 \% d_{n p}=20 \mathrm{~nm}$ because the behavior of the Nusselt number is the same for all PPI, $\phi$ and $d_{n p}$ values.

From Figure 3(a), it is observed as the average Nusselt number increases with $L_{m f} / L_{h w}$ increasing, for Peclet values equal to 750 and 1500, while for $P e$ equal to 350 the trend is opposite. From Figure 3(b), it is possible to observe that the average Nusselt number presents an almost constant value for $L_{m f} / L_{h w}$ values higher than 3.5. 


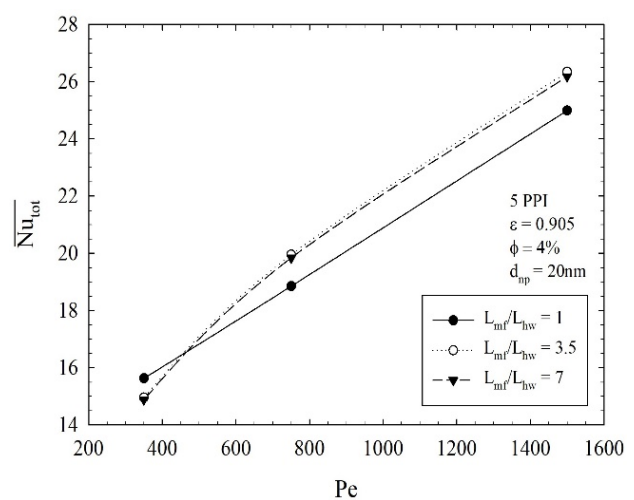

(a)

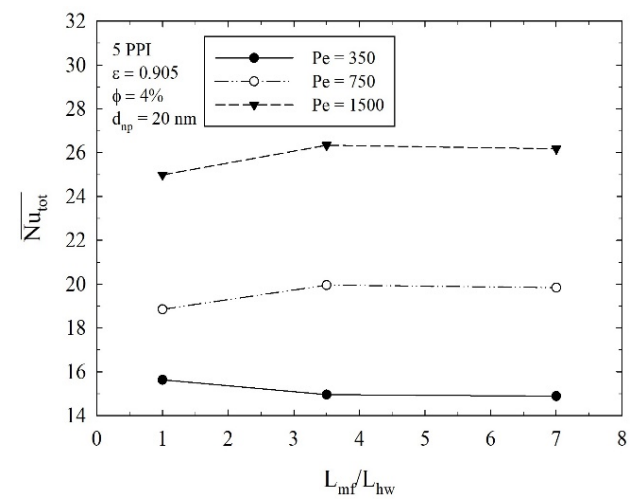

(b)

Fig. 3. Average Nusselt number for 5 PPI, $\phi=4 \%, d_{n p}=80 \mathrm{~nm}$ as a function of (a) Pe number and (b) $L_{m f} / L_{h w}$

In Figure 4(a), the average Nusselt number is showed for different particle volume concentrations $\phi$ for 40 PPI; in Figure 4(b), the average Nusselt number is also showed at varying nanoparticle diameter values. From Figure 4(a), it is observed that $\overline{N u_{t o t}}$ for the pure water is higher than the one for $\phi=1 \%$ and $4 \%$. This happens because the increase in terms of $k_{n f}$ is greater than the one due to the increment of the heat transfer coefficient. Furthermore, from Figure 4(b), it can be noticed that the $\overline{N u_{t o t}}$ decreases with increasing of nanoparticle diameters.

In Figure 5, $\overline{N u_{\text {tot }}}$ is showed at varying PPI values. From Figure 5(a), it can be seen as for Peclet $=350$ the metal foam with 5 PPI is characterized by the higher Nusselt number; when $P e$ increases, $\overline{N u_{t o t}}$ is higher for the metal foam with 20 PPI. The metal foam characterized by 40 PPI and a porosity equal to 0.9132 presents values of average Nusselt number always lower than the metal foam of 5, 10 and 20 PPI that have a porosity equal to 0.90. From Figure 5(b), also at varying PPI, the Nusselt number as a function of $L_{m f} / L_{h w}$ presents its maximum for a value of the ratio between the metal foam length and impinging heated wall length equal to 3.5 .

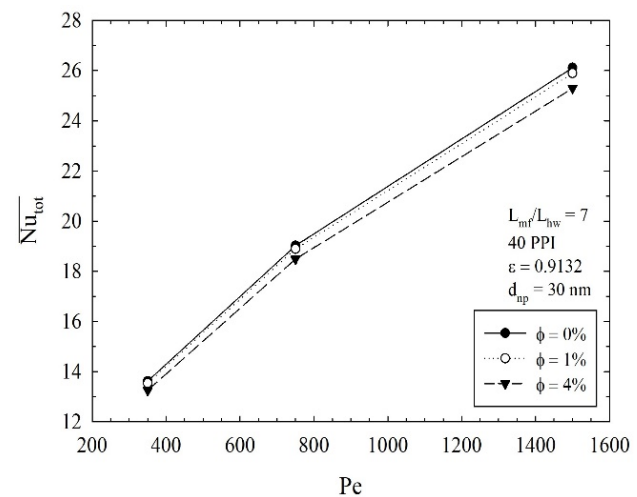

(a)

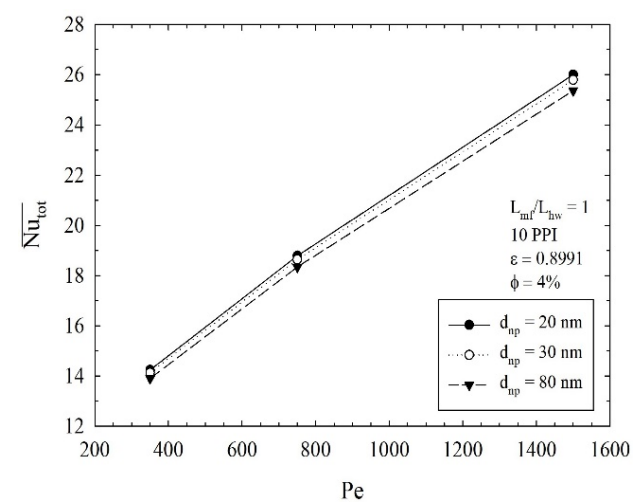

(b)

Fig. 4. Average Nusselt number for (a) 40 PPI, $d_{n p}=30 \mathrm{~nm}$ and different nanoparticle volume concentrations and for (b) 10 PPI, $\phi=4 \%$ and several nanoparticle diameters 


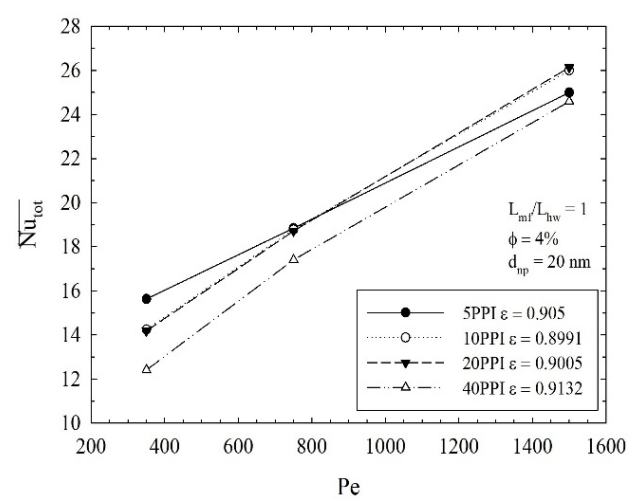

(a)

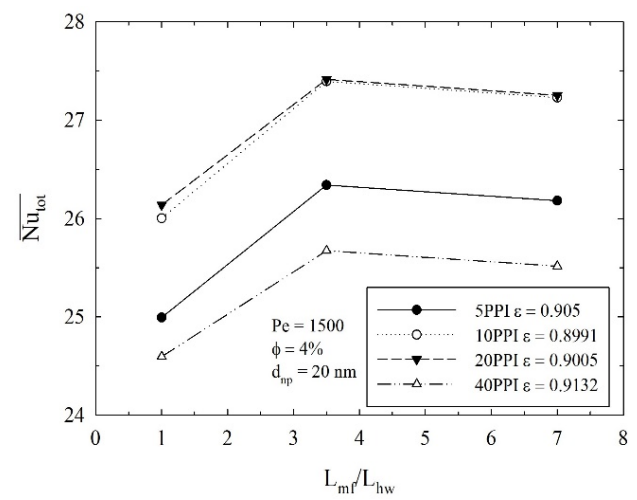

(b)

Fig. 5. Average Nusselt number for $\phi=4 \%, d_{n p}=20 \mathrm{~nm}$ and several PPI as a function of (a) $P e$ number and (b) $L_{m f} / L_{h w}$

In Figure 6, the ratio between the thermal and pumping power is represented for a pore density of 5 PPI, $\phi=4 \% d_{n p}=20 \mathrm{~nm}$. As showed in Figure 6(a), the configuration characterized by the ratio $L_{m f} / L_{h w}$ equal to 1 presents a higher value of $\dot{Q} / \dot{W}$ for all Peclet number values. This means that, when the metal foam length increases, the increase of pressure drop is greater than the heat transfer enhancement. Instead, from Figure 6(b), it is possible to note that the highest $\dot{Q} / \dot{W}$ value is attained for $P e=350$. From the combination of these results, it is possible to deduce that the configuration with the highest efficiency (highest $\dot{Q} / \dot{W}$ ) is the one characterized by the lowest value of $P e$ and $L_{m f} / L_{h w}$ for fixed metal foam properties, nanoparticle volume concentration and nanoparticle diameter.

In Figure 7(a), the ratio between the thermal and pumping power is presented for different particle volume concentrations $\phi$ for 40 PPI and a fixed value of nanoparticle diameter equal to $30 \mathrm{~nm}$; in Figure 7(b), instead, $\dot{Q} / \dot{W}$ is reported for several nanoparticle diameters, at 10 PPI and $\phi=4 \%$. As shown in Figure 7(a), there is a partial overlap between the different analyzed cases especially for low values of $P e$; while, for higher $P e$, it is possible to observe that the ratio $\dot{Q} / \dot{W}$ increases with increasing of $\phi$. Moreover, from Figure 7(b), it is observed that the results in terms of the ratio between the heat transfer rate and the pumping power show an overlap for different nanoparticle diameters. Moreover,

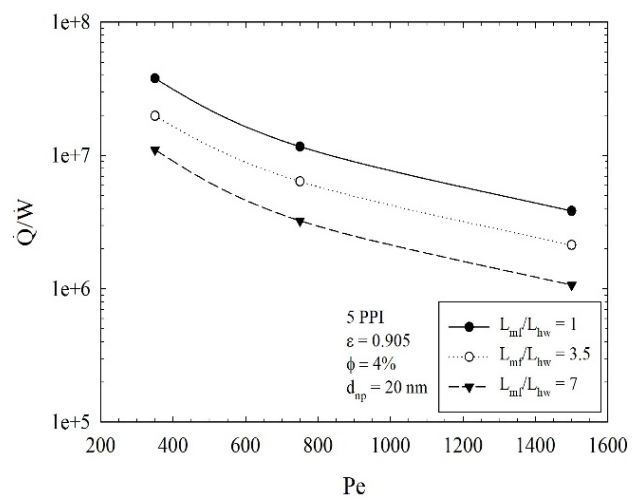

(a)

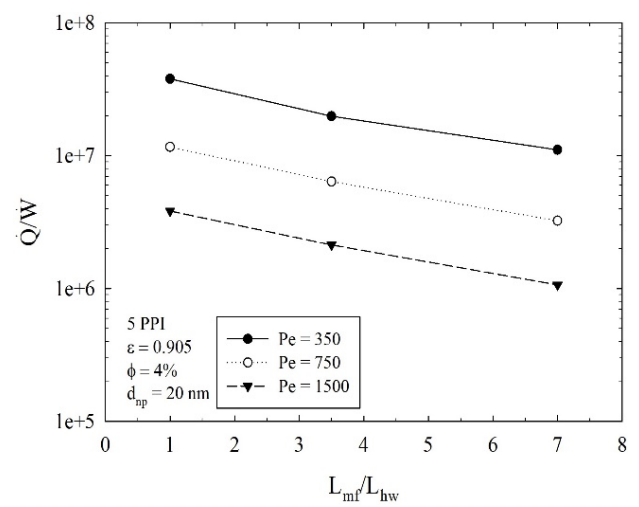

(b)

Fig. 6. $\dot{Q} / \dot{W}$ for 5 PPI, $\phi=4 \%, d_{n p}=80 \mathrm{~nm}$ as a function of (a) $P e$ number and (b) $L_{m f} / L_{h w}$ 


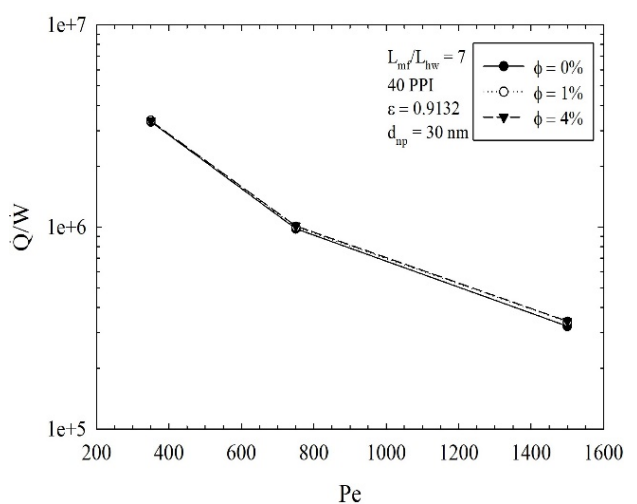

(a)

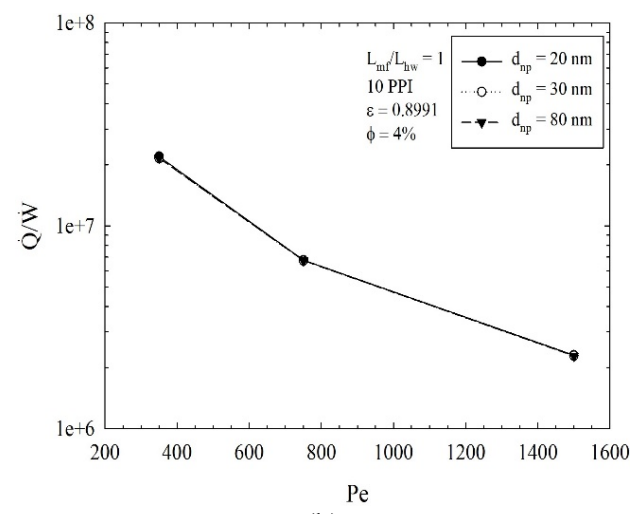

(b)

Fig. 7. $\dot{Q} / \dot{W}$ for (a) 40 PPI, $d_{n p}=30 \mathrm{~nm}$ and different nanoparticle volume concentrations and for (b) 10 PPI, $\phi=4 \%$ and several nanoparticle diameters

it is possible to note that the ratio $\dot{Q} / \dot{W}$ decreases with the increase of Peclet number.

In conclusion, in Figure 8, the ratio $\dot{Q} / \dot{W}$ is showed at varying PPI values. In Figure $8(a)$, the higher ratio between he thermal and pumping power is obtained for the metal foam characterized by 5 PPI and for $P e=350$. In addition, it is possible to observe that $\dot{Q} / \dot{W}$ decreases with the increase of Peclet number. The metal foam characterized by 40 PPI and a porosity equal to 0.9132 presents values of $\dot{Q} / \dot{W}$ always lower than the metal foam with 5, 10 and 20 PPI that have a porosity of 0.90 . From Figure 8 (b), the ratio $\dot{Q} / \dot{W}$ decreases with the increment of $L_{m f} / L_{h w}$ but it assumes the higher value always in corresponding to the metal foam with 5 PPI. In conclusion, from the combination between these data, it is possible to understand that the best compromise between the increase both the heat transfer and the pressure drop is obtained for a length of metal foam equal to the heated wall length $\left(L_{m f} / L_{h w}=1\right)$ and in corresponding to an aluminum foam with 5 PPI and porosity equal to 0.905 .

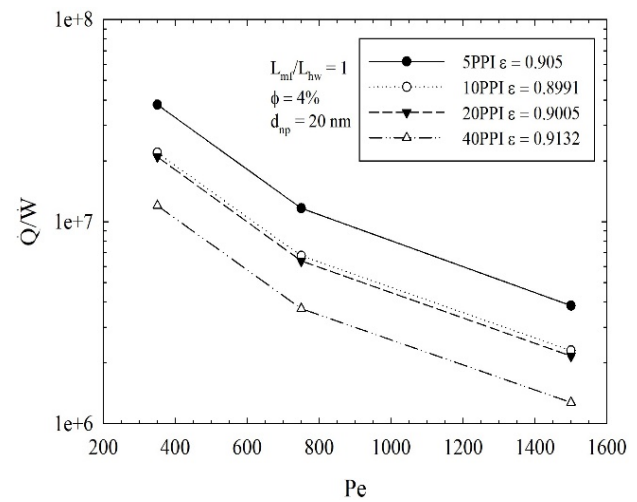

(a)

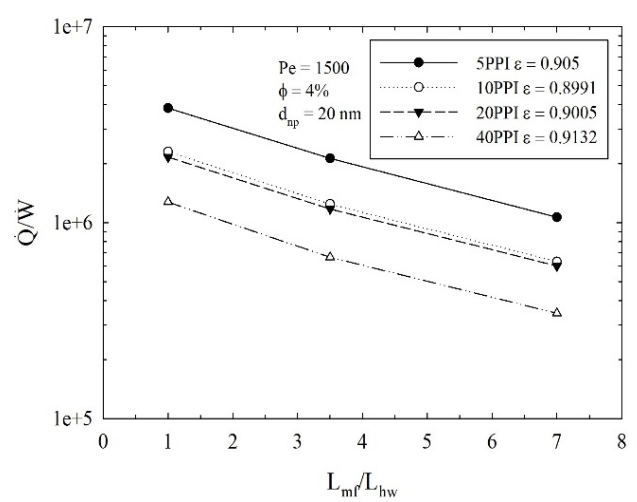

(b)

Fig. 8. $\dot{Q} / \dot{W}$ for $\phi=4 \%, d_{n p}=20 \mathrm{~nm}$ and several PPI as a function of (a) $P e$ number and (b) $L_{m f} / L_{h w}$ 


\section{Conclusions}

In this work a numerical analysis on mixed convection in a confined impinging slot jet with a partially filled configuration of metal foam using $\mathrm{Al}_{2} \mathrm{O}_{3} /$ water based nanofluids is presented. The particle volume concentration ranges from $0 \%$ to $4 \%$ and the particle diameter is equal to 20,30 and $80 \mathrm{~nm}$. The employed metal foams are characterized by a number of pores per inch (PPI) equal to 5, 10, 20 and 40 and a porosity around 0.90 . Results show that the average Nusselt number increases with increasing of $L_{m f} / L_{h w}$ for higher Peclet values while for lower $P e$ the behavior is opposite. Furthermore, $\overline{N u_{t o t}}$ is higher for the pure water respect to $\phi=1 \%$ and $4 \%$ and decreases with increasing of nanoparticle diameters. In addition, the data demonstrate that the higher Nusselt number is presented for metal foam with 5 PPI when Peclet number is low, while, when $P e$ increases $\overline{N u_{t o t}}$ is higher for the metal foam with 20 PPI. From the results in terms of $\dot{Q} / \dot{W}$, it is possible to understand that the configuration with the best compromise between the increase of heat transfer and pressure drop is characterized by the lowest value of $L_{m f} / L_{h w}$ and $P e$ for fixed properties of metal foam and for fixed nanoparticle volume concentration and diameter. Instead, if the properties of metal foam change, the best configuration is obtained in corresponding to an aluminum foam with 5 PPI and porosity equal to 0.905 .

\section{Nomenclature}

\begin{tabular}{|c|c|}
\hline$C_{F}$ & drag factor coefficient \\
\hline$c_{p}$ & specific heat, (J/ kgK) \\
\hline$d_{f}$ & fiber diameter, $(\mathrm{m})$ \\
\hline$d_{n p}$ & nanoparticles diameter, $(\mathrm{m})$ \\
\hline$d_{p}$ & pore diameter, $(\mathrm{m})$ \\
\hline$h$ & heat transfer coefficient, $\left(\mathrm{W} / \mathrm{m}^{2} \mathrm{~K}\right)$ \\
\hline$h_{s f}$ & interfacial heat transfer coefficient, $\left(\mathrm{W} \mathrm{\textrm {m } ^ { 2 } \mathrm { K } )}\right.$ \\
\hline$H$ & system height, $(\mathrm{m})$ \\
\hline$k$ & thermal conductivity, $(\mathrm{W} / \mathrm{mK})$ \\
\hline$K$ & porous permeability, $\left(\mathrm{m}^{2}\right)$ \\
\hline$L$ & system length, $(\mathrm{m})$ \\
\hline$L_{h w}$ & heated wall length, (m) \\
\hline$N u$ & Nusselt number \\
\hline$p$ & pressure, $(\mathrm{Pa})$ \\
\hline$P e$ & Peclet number \\
\hline PPI & number of pores per inch \\
\hline $\operatorname{Pr}$ & Prandtl number \\
\hline$P e$ & Peclet number \\
\hline$\dot{Q}$ & thermal power, $(\mathrm{W})$ \\
\hline $\operatorname{Re}$ & Reynolds number \\
\hline$T$ & Temperature, $(\mathrm{K})$ \\
\hline$u, v$ & $x$ and $y$ velocity components, $(\mathrm{m} / \mathrm{s})$ \\
\hline$W$ & jet width, (m) \\
\hline$\dot{W}$ & pumping power, $(\mathrm{W})$ \\
\hline$x, y$ & Cartesian coordinates, $(\mathrm{m})$ \\
\hline Gre & \\
\hline$\alpha$ & particle Biot number \\
\hline$\beta$ & thermal expansion coefficient \\
\hline
\end{tabular}




$\begin{array}{ll}\alpha_{s f} & \text { specific surface area density, }(1 / \mathrm{m}) \\ \varepsilon & \text { porosity } \\ \mu & \text { dynamic viscosity, }(\mathrm{kg} / \mathrm{ms}) \\ \rho & \text { density, }(\mathrm{kg} / \mathrm{m}) \\ \phi & \text { nanoparticle volume concentration } \\ \text { Subscripts } & \\ b f & \text { base fluid } \\ d_{f} & \text { fiber diameter } \\ m f & \text { metal foam } \\ n f & \text { nanofluid } \\ n p & \text { nanoparticle } \\ s & \text { solid phase of metal foam }\end{array}$

\section{Acknowledgments}

This research was funded by MIUR (Ministero dell'Istruzione, dell'Università e della Ricerca), grant number PRIN-2017F7KZW.

\section{References}

1. M. Amjadian, H. Safarzadeh, M. Bahiraei, S. Nazari, B. Jaberi, Int. Comm. Heat Mass Transf. 112, 104509 (2020).

2. A. Datta, S. Kumar, P. Halder, J. Therm. Sc. 29, 182-193 (2020).

3. I. K. Alabdaly, M. A. Ahmed, Propulsion and Power Research 8 (4), 351-361 (2019).

4. O. Abdelrehim, A. Khater, A. A. Mohamad, A. Radwan, Case Studies Therm.Eng. 14, 100423 (2019).

5. B. Sun, Y. Zhang, D. Yang, H. Li, Appl. Ther. Eng. 151, 556-566 (2019).

6. A. Izadi, M. Siavashi, H. Rasam, Q. Xiong, Appl. Therm. Eng. 168, 114843 (2020).

7. A. Izadi, M. Siavashi, Q. Xiong, Int. J. Hydrogen Energy 44 (30), 15933-15948 (2019).

8. M. Siavashi, H. Rasam, A. Izadi, J. Therm. Analysis and Calorimetry, 135 (2), 13991415 (2019).

9. D. A. Nield, A. Bejan, A., "Convection in Porous Media", 4th Edition, Springer, New York, 2013.

10. S. Whitaker, "The Method of Volume Averaging”, Springer, Netherlands, 1998.

11. A: Bhattacharya, V. V. Calmidi, R. L. Mahajan, Int. J. Heat Mass Transf. 45, 10171031 (2001).

12. V. V. Calmidi, R. L. Mahajan. ASME J. Heat Transf. 122, 557-565 (2000).

13. K. Khanafer, K. Vafai, M. Lightstone, J. Heat Mass Transf. 46 (19), 3639-3653 (2003).

14. G. Sekrani, S. Poncet, P. Proulx, Chemical Eng. Sc. 176, 205-219 (2018).

15. B. C. Pak, Y. L. Cho, Exp. Heat Transf. Int. J. 11 (2), 151-170 (1998).

16. C. W. Nan, R. Birringer, D. R. Clarke, H. Gleiter, J. Appl. Physics, 81 (10), 66926699 (1997). 